\title{
Consolidação dos Sistemas de Gestão nas Empresas: Análise da Implantação dos Sistemas de Gestão Ambiental no Brasil
}

\author{
Consolidation of Management Systems in Companies: Analysis of the \\ Implementation of Environmental Management Systems in Brazil
}

\author{
Herlander Mata-Lima ${ }^{1}$ \\ Flaviana Nunes ${ }^{2}$ \\ Andreilcy Alvino-Borba ${ }^{3}$ \\ José António Almeida ${ }^{4}$
}

\section{Resumo}

\begin{abstract}
No âmbito deste trabalho foram avaliados: (1) o progresso relativo à implantação do sistema de gestão ambiental (SGA) no seio empresarial, comparativamente ao sistema de Gestão da Qualidade (SGQ); (2) a importância relativa dos sistemas de gestão em função dos setores de atividade econômica; e (3) os aspectos e impactos ambientais dominantes no setor com maior número de organizações certificadas. As pesquisas incidiram sobre a base de dados internacional (Scopus) para verificar a tendência evolutiva das publicações regionais, e a análise da evolução de organizações certificadas se baseou numa amostra de 180 organizações extraídas da base de dados do INMETRO. A identificação e a avaliação dos aspectos e impactos ambientais das organizações e as respectivas medidas mitigadoras resultaram da análise das atividades, produtos e serviços referentes ao setor de atividade econômica na qual estão enquadradas. Os resultados indicaram que o maior número de organizações certificadas estão localizadas em São Paulo (estado com maior densidade industrial) e que o setor com maior número de empresas certificadas tem como atividade a transformação industrial. Também se constatou que os aspectos ambientais desse setor são significativos porque afetam todos os componentes ambientais (água, atmosfera, solo e biota), situação que requer o monitoramento dos indicadores ambientais na região sob influência dessas empresas, como condição essencial para tomada de decisão no domínio da conservação dos serviços de ecossistemas.
\end{abstract}

Palavra-chave: Gestão Ambiental; ISO 14001; Aspectos Ambientais; Mitigação dos Impactos; Sustentabilidade Ambiental.

1 CERENA - Instituto Superior Técnico, Universidade de Lisboa. Av. Rovisco Pais, 1. 1049-001, Lisbon, Portugal PROAC - Universidade Federal de Juíz de Fora (UFJF), Juiz de Fora-Minas Gerais, Brasil

2 Universidade Federal do ABC, Santo André, São Paulo, Brasil

3 Doutoranda na Universidade Aberta de Lisboa, Lisboa, Portugal

4 GeoBioTec, FCT/UNL - Faculdade de Ciências e Tecnologia, Universidade Nova de Lisboa, Lisboa, Portugal 


\section{Abstract}

This work evaluated (1) the progress on the implementation of the environmental management system (EMS) compared to the Quality Management System (QMS), (2) the relative importance of the management systems according to sectors of economic activity and (3) aspects and prevailing environmental impacts in the sector with the largest number of certified organizations. The research focused on the international database (Scopus) to verify the evolutionary trend of regional publications and the analysis of the evolution of certified organizations was based on a sample of 180 organizations drawn from INMETRO database. The identification and evaluation of environmental aspects and impacts of the organizations and their mitigation measures resulted from the analysis of the activities, products and services for the economic sector in which they are framed. The results indicated that the largest number of certified organizations is located in São Paulo (state with the highest industrial density), the sector with the largest number of certified companies is engaged in the industrial processing. It was also found that the environmental aspects of industry are significant because they affect all environmental components (water, air, soil and biota), a situation which requires the monitoring of environmental indicators in the region under the influence of these companies as essential for decision making concerning ecosystem services of conservation.

Keywords: Environmental Management; ISO 14001; Environmental Aspects; Mitigation of Impacts; Sustainability.

\section{Introdução}

Um dos grandes desafios da gestão ambiental diz respeito ao planejamento do território, pois exige o domínio dos subsistemas que compõem o sistema biofísico (atmosfera, hidrosfera e litosfera) e a interação entre eles (LOISEAU et al., 2012). A combinação das condições de ocupação e uso do solo, no presente, com a projeção da evolução do crescimento demofórico, permite diagnosticar as condições ambientais atuais e planejar preventivamente o desenvolvimento socioeconômico.

Dentre as atividades que mais contribuem para poluição do ambiente urbano, destacamse o tráfego de automóvel, a atividade industrial e o aumento da densidade de infraestrutura (GRIGORATOS et al., 2014). Os impactos de tais atividades se transmitem para a dimensão socioeconômica afetando os objetivos do desenvolvimento sustentável que pretende "satisfazer as necessidades humanas conservando a capacidade de os sistemas da terra suportarem a vida e reduzindo a fome e a pobreza”.

Os sistemas de gestão da qualidade, do ambiente e da segurança no trabalho conduzem a diversas vantagens competitivas para as empresas, tais como: (1) diminuição do desperdício de matéria-prima e energia; (2) diminuição de desconformidades que se traduz na eficiência produtiva; (3) prolongamento do ciclo de vida dos produtos que viabiliza a diminuição do volume de rejeitos e custos de tratamento e disposição final; (4) boas práticas na gestão de resíduos priorizando redução, reutilização, reciclagem e incorporação de tecnologias avançadas no tratamento de efluentes líquidos . Por outro lado, o sistema de gestão ambiental garante a coleta de dados que 
permitem quantificar os indicadores para o monitoramento e avaliação de desempenho ambiental do sistema produtivo nas empresas (TESTA et al., 2014).

Tradicionalmente, regiões com maior crescimento econômico possuem maior número de organizações industriais (SU et al., 2013; NEAL et al., 1997) e, por consequência, exibem aspectos e impactos ambientais mais significativos que se manifestam em elevados índices de pegada de carbono, hídrica e ecológica (SILVA et al., 2015).

Este estudo foi realizado com intuito de (1) caracterizar a tendência evolutiva da gestão ambiental nas empresas através da análise espacial e temporal das certificações atribuídas, identificar os setores de atividade econômica com maior número de empresas certificadas, (2) derivar os aspectos e impactos ambientais para a atividade econômica dominante com o objetivo de entender as relações com a degradação dos serviços do ecossistema e (3) propor medidas para mitigar os impactos ambientais significativos.

\section{Área de Estudo}

Localizado na região sudeste, São Paulo é o maior estado brasileiro e acolhe uma população total de aproximadamente 41,2 milhões de habitantes (Tabela 1). É, também, o estado que mais contribui para o produto interno bruto (PIB) em termos absolutos, pois possui um índice de densidade industrial que o coloca em destaque no grupo superior, conjuntamente com Rio de Janeiro e Rio Grande do Sul (SABOIA, 2013, p. 239, 244).

Tabela 1. Estados brasileiros com maior PIB per capita

\begin{tabular}{|c|c|c|c|c|c|c|}
\hline \multirow[b]{2}{*}{ Estado } & \multirow{2}{*}{$\begin{array}{l}\text { Populaçãoo } \\
(\text { hab) }\end{array}$} & \multirow{2}{*}{$\underset{\mathrm{R} \$)}{\operatorname{PIB}\left(10^{6}\right.}$} & \multicolumn{2}{|c|}{ PIB per capita } & \multicolumn{2}{|c|}{ Empresas $^{c}$} \\
\hline & & & $\mathbf{R} \$$ & USD\$ & Total & $\begin{array}{c}\text { Posição } \\
\text { Nacional }\end{array}$ \\
\hline Distrito Federal (DF) & 2.570 .160 & 129.634 & 50.438 & 22.574 & 225.678 & 14 \\
\hline São Paulo (SP) & 41.262.199 & 1.084 .866 & 26.292 & 11.767 & 3.782 .075 & 1 \\
\hline Rio de Janeiro (RJ) & 15.989 .929 & 353.425 & 22.103 & 9893 & 1.054 .988 & 3 \\
\hline Santa Catarina (SC) & 6.248 .436 & 132.561 & 21.215 & 9495 & 555.006 & 7 \\
\hline
\end{tabular}

Fonte: a IBGE, Censo Demográfico 2010; b IBGE, Contas regionais do Brasil 2009; ' Instituto Brasileiro de Planejamento Tributário (IBPT) - Censo das Empresas e Entidades Públicas e Privadas Brasileiras - Empresômetro 2012

A Região Metropolitana de São Paulo (RMSP) enfrenta todos os problemas ambientais e sociais - consequentes da elevada densidade populacional, industrial e de área construída - típicos das grandes metrópoles das regiões em vias de desenvolvimento. Tais problemas dividem-se nos cinco seguintes grupos: (i) a excessiva densidade de tráfego aéreo e terrestre no espaço urbano contribui para elevados níveis de poluição atmosférica, psicológica (estresse resultante da dificuldade de mobilidade urbana) e sonora (BRUNELLE-YEUNG et al., 2014, BARCZAK; DUARTE, 2012); (ii) existência de muitas áreas de solos poluídos devido à ausência de controle e recuperação de brownfield (terrenos previamente usados para propósitos industriais e/ou comerciais) (GÜNTHER, 2006); (iii) a região não possui um único rio que não esteja poluído por efluentes domésticos/industriais e resíduos sólidos devido à pressão antrópica (TUCCI, 2008); (iv) os Resíduos Sólidos Urbanos (RSU) ainda não são adequadamente geridos numa perspectiva de redução, reuso (aumento do ciclo de vida) e reciclagem. Não obstante as melhorias verificadas nos últimos anos, a maior parte de resíduos ainda não tem como destino final aterros 
controlados porquanto a maior parte (60\%, segundo LEME et al., 2014) dos 60,7 milhões de toneladas de resíduos produzidos anualmente ainda são despejados em lixões a céu aberto (OMAR et al., 2012, JACOBI; BESEN, 2011, TUCCI, 2008); (v) os problemas anteriormente descritos desencadeiam elevada incidência de doenças transmitidas por vetores presentes na atmosfera, água e solo causando diversas enfermidades incluindo as psíquicas (HAIDER; HASHMI, 2014, KONDO et al., 2014, REHFUESS; BARTRAM, 2014, MCKENZIE et al., 2014).

\section{Procedimento Metodológico}

O procedimento metodológico adotado considerou três etapas distintas: na primeira, efetuou-se o levantamento de dados referentes a evolução da pesquisa, medida em termos do número de publicações nas área da gestão ambiental (ISO 14001) e qualidade (ISO 9001) na base de dados internacional Scopus, com o intuito de verificar as tendências em termos globais e regionais. Foram apenas considerados artigos publicados em periódicos até 2014;na segunda etapa, foram analisados os dados correspondentes às empresas certificadas. Os dados provieram de uma plataforma desenvolvida pelo Instituto Nacional de Metrologia, Qualidade e Tecnologia (INMETRO, http://www.inmetro.gov.br/credenciamento/) e ABNT/CB-25, objetivando caracterizar a evolução agregada e distribuída por setor de atividade econômica (em função do código NACE - Nomenclatura estadística de atividade econômica) no que concerne à atribuição das certificações aos sistemas de gestão implantados nas organizações. Os resultados foram construídos com base em uma amostra de 180 empresas certificadas, em diferentes setores;

a última etapa consistiu na descrição dos aspectos ambientais (i.e. elementos de atividades, produtos e serviços da empresa que possam interagir com o ambiente) e os impactos decorrentes para o setor de atividade econômica com maior prevalência de empresas certificadas pela ISO 14001. Em seguida, descreveram-se as medidas de mitigação para os potenciais impactos dos aspectos identificados.

\section{Resultados e Discussões}

\section{Evolução da Produção Científica}

O levantamento das publicações relacionadas ao tema "ISO 14001", efetuado em março de 2014, na base de dados da Scopus devolveu o resultado de 1370 registros, no período de 1993 a 2014. Também realizaram buscas com a expressão "ISO 9001", sendo os resultados sintetizados na Figura 1, em termos anuais e regionais.

A Figura 1a ilustra que, até o ano de 1995, não houve publicações sobre o tema "ISO 14001", mas, a partir de 1999, o número de publicações anuais situou-se sempre acima de 60. A Figura 1 b revela que o Brasil está entre os cinco (5) países onde a comunidade técnico-científica dedica maior atenção ao tema. Relativamente à ISO 9001, a situação internacional (global) é semelhante àquela verificada para ISO 14001, mas Brasil não se encontra no top 10 como se constata nas Figura 1c, d. 


\section{Figura 1. Evolução das publicações sobre SGA (14001) e SGQ (9001) no período de 1987 a 2014}

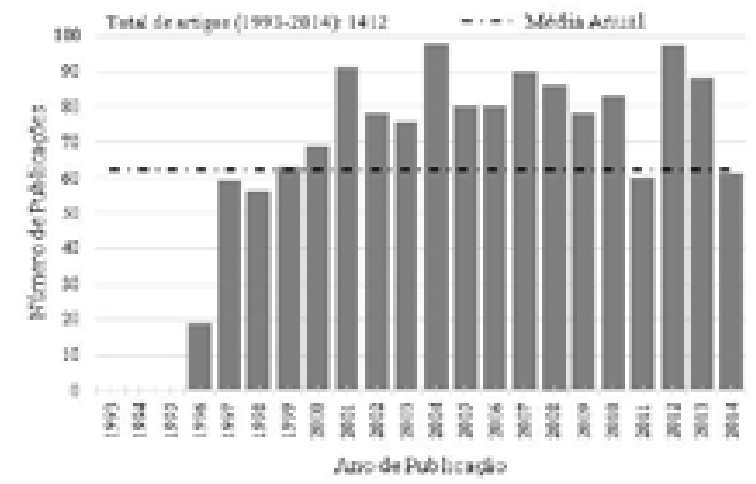

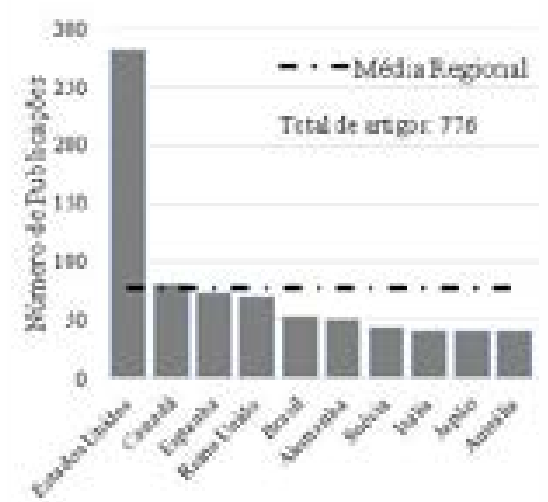

Regiss se Dublisatso dep 10 (a) Base de dados Scopus - ISO 14001

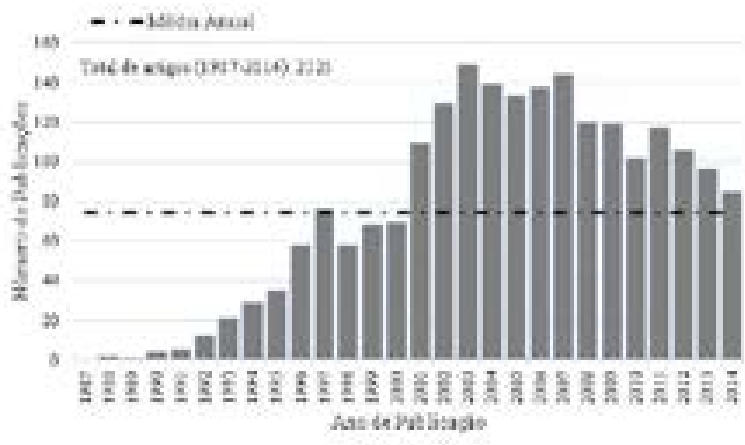

(c) Base de dados Scopus - ISO 9001 (b) Scopus pesquisa regional - ISO 14001

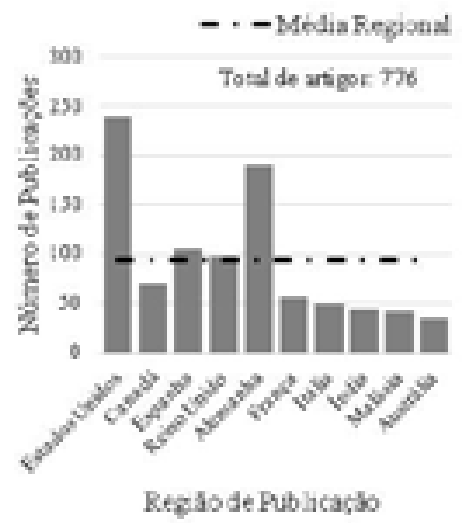

(d) Scopus pesquisa regional - ISO 9001

As publicações sobre o "ISO 9001" surgiram no início da década de 80, i.e. sensivelmente uma década antes das da ISO 14001. A maior ascensão ocorreu nos anos 1990 que corresponde ao período de desenvolvimento e consolidação das normas ISO 9001 e 14001 . O fato de existir maior produção de publicações referentes ao tema "ISO 9001" deve-se ao fato da qualidade estar intrinsecamente ligada ao desempenho produtivo das empresas enquanto que as questões ambientais não têm sido associadas a oportunidade de melhorar continuamente o processo produtivo. Esta situação configura também um baixo (quase inexistente) nível de compromisso relativamente às questões relacionadas à responsabilidade social - conforme a norma ISO 26000 -, tais como transparência, comportamento ético, prestação de contas, respeito aos interesses dos stakeholders, respeito à lei, respeito às normas internacionais e respeitos pelos direitos humanos (vide, e.g., ÁVILA et al., 2013).

\section{Empresas Certificadas: Gestão Ambiental vs Gestão da Qualidade}

A base de dados do INMETRO ainda não está devidamente atualizada para disponibilizar os dados sobre todas as empresas certificadas. Nesse contexto, o estudo se baseia numa amostra de 180 empresas certificadas pela ISO 14001 e 3461 empresas certificadas pela ISO 9001. 
Quanto à certificação ISO 14001, constatou-se que do total das empresas contidas na amostra, 64 se localizam no estado de São Paulo e 10 no Rio de Janeiro. Merece ainda destaque que das 64 organizações de São Paulo, 30 estão situadas na Região Metropolitana de São Paulo (RMSP), conforme a Figura 2.
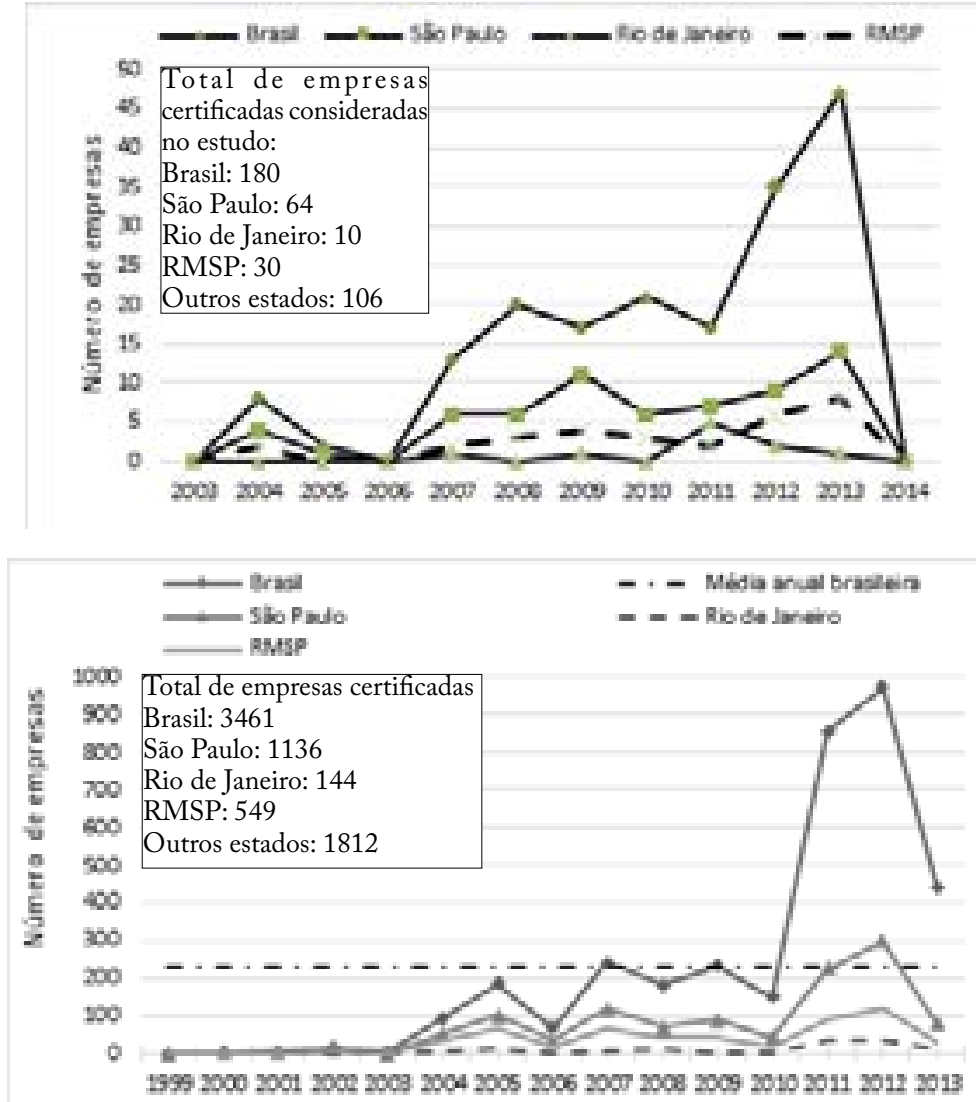

O número de certificações atribuídas no período de 2011 a 2013 corresponde ao dobro da média anual das certificações em todo o período considerado. Contudo, o número de certificações na RMSP permaneceu sempre abaixo de 10 empresas/ano, situação que indica que o significativo incremento global no período de 2011 a 2013 se deve ao crescimento de certificações em outras regiões do país. Rio de Janeiro sofreu redução significativa ( 83\%) do número de certificações anuais no período de 2011 a 2013. No que diz respeito às empresas certificadas pela ISO 9001, observou-se que do total de 3461 empresas certificadas, 1136 e 144 estão situadas no estado de São Paulo e Rio de Janeiro, respectivamente. Dentre as empresas certificadas no estado de São Paulo 48\% estão inseridas na RMSP (vide Figura 2b).

A figura 2 ilustra que na base de dados do INMETRO não existem dados para os anos de 2003 e anteriores, mas os responsáveis do INMETRO informaram que se encontra atualmente em andamento um plano de ação para manter a base de dados atualizada.

À semelhança da ISO 14001, também se observa um aumento significativo das certificações ISO 9001 entre 2010 e 2011, tendo atingido um número mais de três vezes superior à média anual das certificações, como consequência do aumento significativo do número de empresas que obtiveram certificação noutras regiões do país visto que não houve aumento muito significativo em São Paulo e Rio de janeiro.

Segundo dados do Instituto Brasileiro de Planejamento Tributário (IBPT), em 2012, o estado de São Paulo possuía 3.782.075 organizações (públicas e privadas) e Rio de Janeiro ocupava a segunda posição com 1.054 .988 ( 28\% do total das organizações existentes em São Paulo). 


\section{Análise Distribuída da Certificação ISO 14001}

Apresenta-se uma análise distribuída das certificações em função das unidades federativas do país e dos setores de atividade econômica (código NACE), incluindo as subcategorias inseridas no setor principal.

A Figura 3 resume a análise referente à distribuição espacial das empresas certificadas no território nacional.

\section{Figura 3- Empresas com certificação ISO 14001, no período de 2004 a 2013, em} função das unidades federativas brasileiras

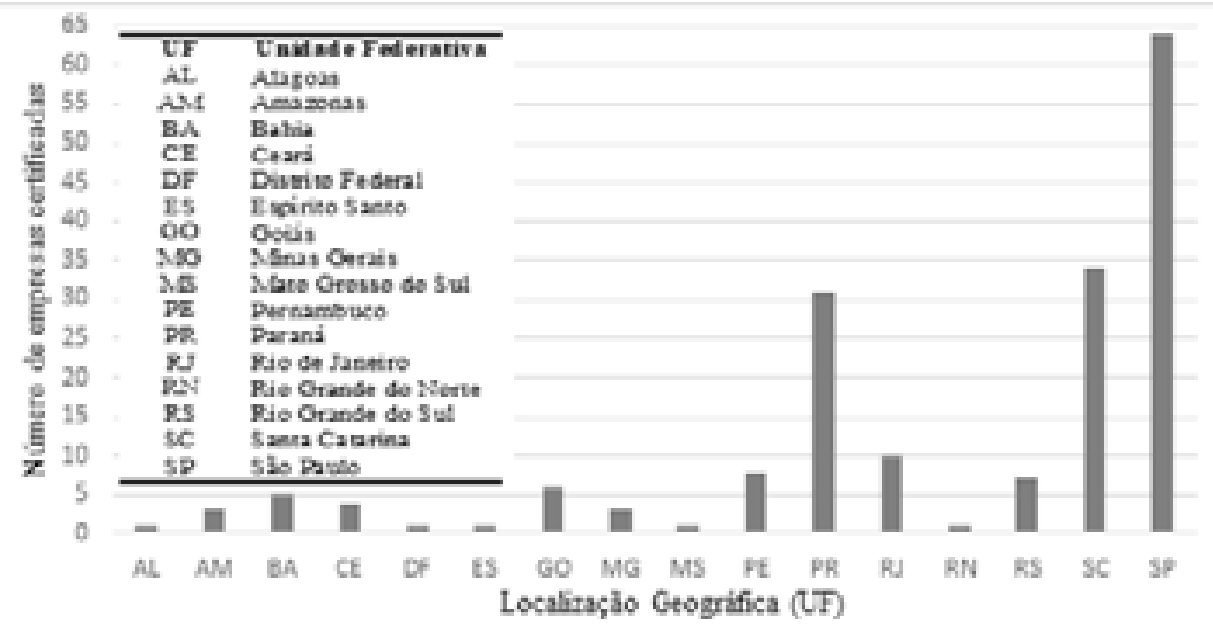

Observa-se que o estado de São Paulo, no contexto da amostra analisada, tem o maior número de empresas certificadas, totalizando 35,5\%, seguido por Santa Catarina e Paraná com $18,9 \%$ e $17,2 \%$ de empresas certificadas, respectivamente.

Considerando que o estado de São Paulo está subdividido em 5 regiões metropolitanas, elaborou-se a Figura 4 que mostra a quantidade de empresas com certificação ISO 14001 nas regiões metropolitanas de São Paulo.

\section{Figura 4 - Empresas com certificação ISO 14001 nas regiões metropolitanas do estado de São Paulo}

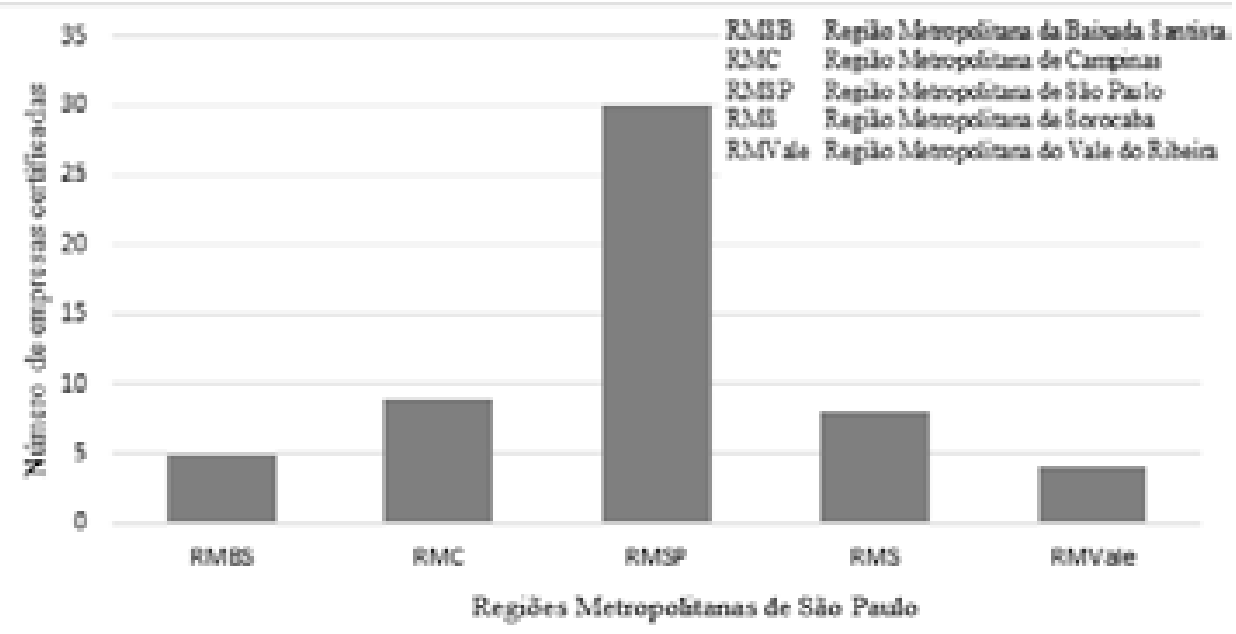


A RMSP possui maior quantidade (47\%) de organizações certificadas, comparativamente às restantes regiões metropolitanas do estado. Importa ressaltar que determinadas cidades do estado de São Paulo (Sorocaba, Bragança Paulista, Jundiaí, etc.) não se encontram incluídas em regiões metropolitanas, pois constituem aglomerações urbanas 'subordinadas' a um polo administrativo.

Os municípios que compõem a RMSP apresentam grande diversidade de atividades econômicas que possuem aspectos e impactos ambientais que carecem de identificação e tratamento como condição indispensável para garantir o devido planejamento e gestão territorial. De outro modo, os serviços dos ecossistemas (WANG et al., 2014, CROSSMAN et al., 2013) ficam comprometidos manifestando-se através de impactos ambientais e socioeconômicos a escala local e regional.

Apesar de ter uma porcentagem (47\%) superior de certificações comparativamente às outras unidades federativas, a situação ainda não é favorável devido ao fato de ser o estado do país com organizações de maior intensidade tecnológica e, consequentemente, com mais aspectos e impactos ambientais significativos. Neste trabalho, houve a preocupação de listar as atividades econômicas para viabilizar a análise dos aspectos e impactos ambientais, assim como das medidas de mitigação mais adequadas para cada caso - conforme se apresenta na seção seguinte (dedicada aos Aspectos e Impactos Ambientais).

Elaborou-se uma base de dados com os itens relevantes para o projeto, de modo a filtrar dados mais específicos das empresas, conforme se descreve a seguir. A discretização por setor de atividade, incluída na Figura 5, refere-se aos setores de atividade econômica das empresas certificadas pela ISO 14001 em nível nacional.

\section{Figura 5 - Empresas certificadas pela ISO 14001, no período de 2004 a 2013 $v s$ atividade econômica (Código NACE - Nomenclatura estatística de atividade econômica)}

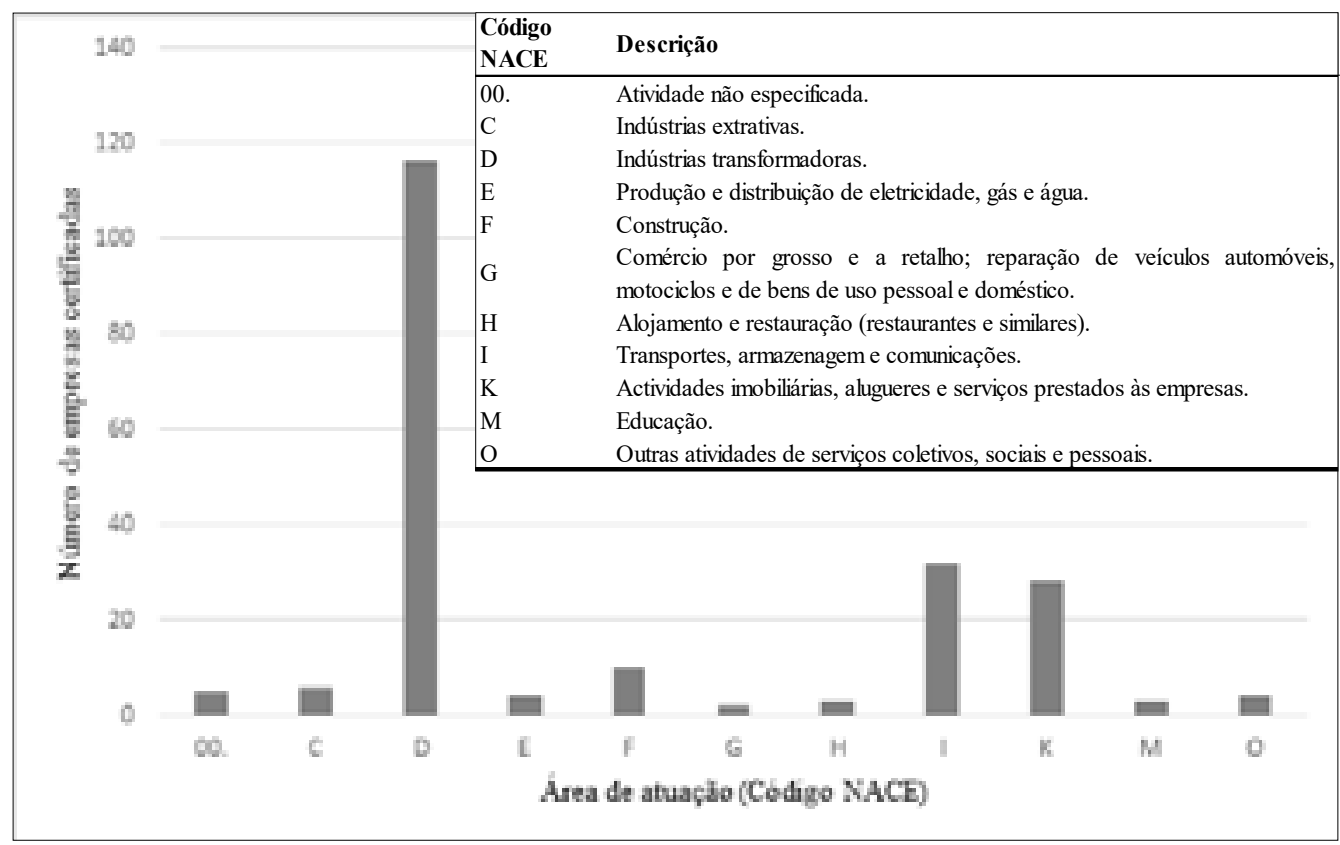

Constata-se, através da figura 5, que a grande maioria das empresas, na amostra considerada (i.e., cento e dezesseis) - pertence à categoria $\mathbf{D}$ (indústrias transformadoras) que engloba catorze (14) subcategorias relacionadas à indústria de transformação. $\mathrm{Na}$ ordem decrescente de 
importância, surgem as categorias I (transporte, armazenagem e comunicações) e K (atividades imobiliárias, alugueres e serviços prestados às empresas) com, respectivamente trinta e duas (32) e vinte e oito (28) empresas certificadas.

Considerando que a Figura 5 ressalta que as indústrias transformadoras congregam maior número de certificações ambientais, efetuou-se uma análise desagregada das 14 subcategorias incluídas na categoria $\mathbf{D}$ relativamente à distribuição de organizações certificadas de acordo com a ISO 14001 (Figura 6).

Figura 6. Empresas certificadas pela ISO 14001 (período de 2004 a 2013) vs atividade econômica das subcategorias da categoria $D$ (indústrias transformadoras) do código NACE.

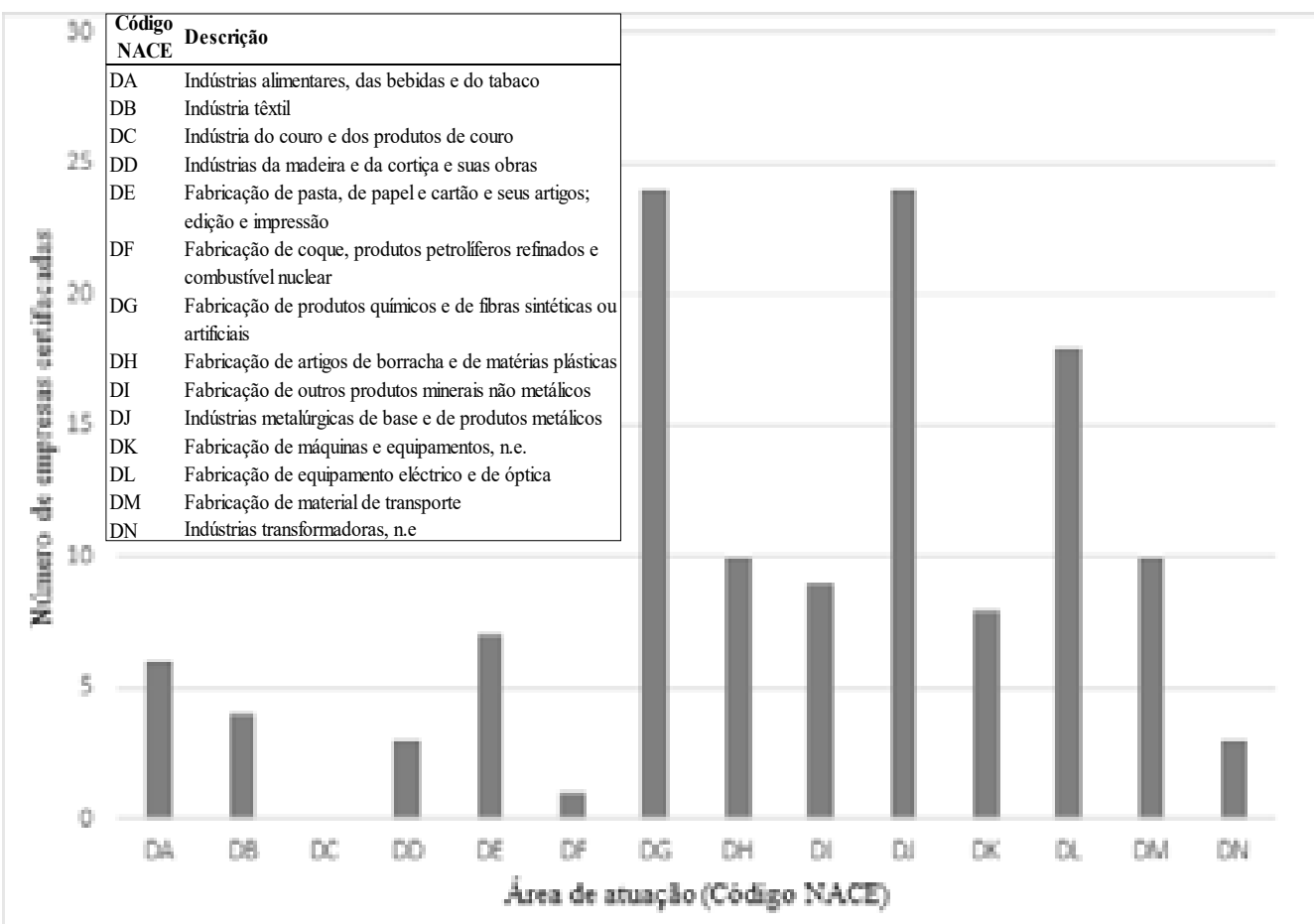

A Figura 6 revela que a maior parte de empresas brasileiras certificadas se concentram nas subcategorias DG (Fabricação de produtos químicos e de fibras sintéticas ou artificiais) e DJ (Indústrias metalúrgicas de base de produtos metálicos) ambas com 24 empresas certificadas, seguidas pela subcategoria DL (Fabricação de equipamentos elétricos e de óptica), com 18 empresas certificadas. De realçar que, de acordo com a informação disponível atualmente na base de dados do INMETRO, a subcategoria DC (Indústrias de couro e produtos de couro) não possui organizações certificadas.

Aspectos e Impactos Ambientais

Esta seção sintetiza a análise das atividades em função (1) dos aspectos e impactos ambientais (vide Tabela 3) e (2) do âmbito da certificação das empresas (vide Tabela 4). Foram inventariados os aspectos e impactos envolvidos e apresentaram-se propostas de medidas mitigadoras condizentes com a estratégia da gestão ambiental enunciada nos princípios da gestão do ambiente (sobretudo os princípios de prevenção, precaução e redução na fonte). 
A esse respeito convém ainda realçar que o estudo realizado por Oliveira et al. (2010, p. 1799), essencialmente baseado na aplicação de um questionário a 69 empresas brasileiras certificadas, identificou as vantagens e desvantagens que as empresas brasileiras entendem que resultam da certificação (Tabela 2 ).

\section{Tabela 2. Vantagens e desvantagens da certificação ambiental segundo empresas brasileiras}

\begin{tabular}{|c|c|}
\hline Vantagens & Desvantagens \\
\hline $\begin{array}{l}\text { - Desenvolvimento de ações ambientais preventivas } \\
\text { - Redução do consumo de energia, água, gás e } \\
\text { combustível } \\
\text { - Influência positiva nos processos de gestão } \\
\text { intraorganizacional. }\end{array}$ & $\begin{array}{l}\text { - Aumento de custos devido à gestão ambiental de } \\
\text { acordo com a ISO } 14001^{*} \\
\text { - Mudanças frequentes na legislação ambiental } \\
\text { brasileira. }\end{array}$ \\
\hline \multicolumn{2}{|c|}{$\begin{array}{l}\text { *Merece destaque o modo como esta desvantagem apontada evidencia o fraco sentido de responsabilidade socia } \\
\text { (vide ISO } 26000 \text { e e consciência ambiental da parte dos gestores das empresas que, ao que tudo indica, continuam } \\
\text { a não internalizar o princípio de poluidor-pagador como forma de garantir a sua própria sustentabilidade a longo } \\
\text { prazo. Por conseguinte, é legítimo afirmar que ainda vigora a linha de pensamento segundo a qual o ecossistema } \\
\text { é capaz de oferecer serviços indefinidamente não obstante o agravamento dos impactos ambientais resultante do } \\
\text { crescimento demofórico. }\end{array}$} \\
\hline
\end{tabular}

A proliferação das análises efetuadas na Tabela 3 e Figura 7 e a apresentada na Tabela 4 permitiria que, uma vez identificado o setor de atividade econômica da empresa e inventariadas as suas atividades laborais, fosse possível compreender a distribuição dos aspectos e impactos ambientais, dominantes nas nossas grandes metrópoles, com o intuito de definir prioridades no que concerne à definição de um plano de ação (regional e local) para mitigar os efeitos ambientais mais significativos e que exigem especial cuidado no contexto do planejamento da ocupação e uso do solo e gestão ambiental. 
Tabela 3. Aspectos e impactos ambientais dos vários setores de atividade econômica abrangidos pelas organizações da amostra analisada (vide Figura 5)

\begin{tabular}{|l|c|c|c|c|c|c|c|c|c|c|}
\hline \multicolumn{1}{|c|}{ Atividades } & A & B & C & D & E & F & G & H & I & J \\
\hline Consumo de energia & $\mathrm{X}$ & $\mathrm{X}$ & $\mathrm{X}$ & $\mathrm{X}$ & $\mathrm{X}$ & $\mathrm{X}$ & $\mathrm{X}$ & $\mathrm{X}$ & $\mathrm{X}$ & $\mathrm{X}$ \\
\hline Geração de resíduos sólidos & $\mathrm{X}$ & $\mathrm{X}$ & $\mathrm{X}$ & $\mathrm{X}$ & $\mathrm{X}$ & $\mathrm{X}$ & $\mathrm{X}$ & $\mathrm{X}$ & & $\mathrm{X}$ \\
\hline Consumo de água & & $\mathrm{X}$ & $\mathrm{X}$ & $\mathrm{X}$ & $\mathrm{X}$ & $\mathrm{X}$ & $\mathrm{X}$ & $\mathrm{X}$ & & $\mathrm{X}$ \\
\hline Emissões gasosas & $\mathrm{X}$ & & $\mathrm{X}$ & $\mathrm{X}$ & $\mathrm{X}$ & $\mathrm{X}$ & $\mathrm{X}$ & & & \\
\hline Geração de ruídos & & $\mathrm{X}$ & & $\mathrm{X}$ & & $\mathrm{X}$ & $\mathrm{X}$ & $\mathrm{X}$ & $\mathrm{X}$ & \\
\hline Lançamento de efluentes & & $\mathrm{X}$ & $\mathrm{X}$ & & $\mathrm{X}$ & $\mathrm{X}$ & $\mathrm{X}$ & & & \\
\hline Geração de águas residuárias & & $\mathrm{X}$ & & $\mathrm{X}$ & & $\mathrm{X}$ & $\mathrm{X}$ & & & \\
\hline Esmissões de partículas ou poeira & & & & $\mathrm{X}$ & & $\mathrm{X}$ & $\mathrm{X}$ & & & \\
\hline Consumo de matérias primas & $\mathrm{X}$ & & $\mathrm{X}$ & & & & & & & \\
\hline Remoção da vegetação natural & & & & & & & & $\mathrm{X}$ & $\mathrm{X}$ & \\
\hline Alteração do trânsito local & & & & & & & & & $\mathrm{X}$ & \\
\hline Armazenamento de produtos perigosos & & $\mathrm{X}$ & & & & & & & & \\
\hline Deposição de escória & & & & $\mathrm{X}$ & & & & & & \\
\hline Geração de lodo & & & & $\mathrm{X}$ & & & & & & \\
\hline Impermeabilização de áreas & & & & & & & & $\mathrm{X}$ & & \\
\hline Terraplanagem & & & & & & & & $\mathrm{X}$ & & \\
\hline Vazamento ou derramamento de matéria-prima, água e óleo & & & $\mathrm{X}$ & & & & & & & \\
\hline
\end{tabular}

Legenda: A - Atividades relacionadas com a impressão; B - Gases industriais; Produtos químicos inorgânicos de base - outros; Tintas, vernizes e similares, tintas de impressão e mástiques; Produtos farmacêuticos e médicohospitalares de base; Produtos químicos - outros; C - Produtos de borracha - outros; Embalagens de plástico; Artigos de plástico - outros; D - Ferro gusa, ferro fundido, aço e ferro-ligas; Laminação à frio de tiras; E - Estruturas metálicas e componentes de estruturas; Peças metálicas forjadas, estampadas, perfiladas; metalurgia do pó; F - Motores e turbinas (exceto motores para aeronaves, automóveis e motocicletas); Fabricação, comercialização, instalação e assistência técnica de aquecedores de água por energia solar e/ou elétrica, e aquecedores de piscinas; Máquinas e equipamentos de uso específico - outros; G - Equipamentos elétricos para motores e veículos, não especificados; H - Serviços gerais de construção de edifícios e de engenharia civil; I - Manuseio de Carga; J Atividades de consultoria dos negócios e de gestão.

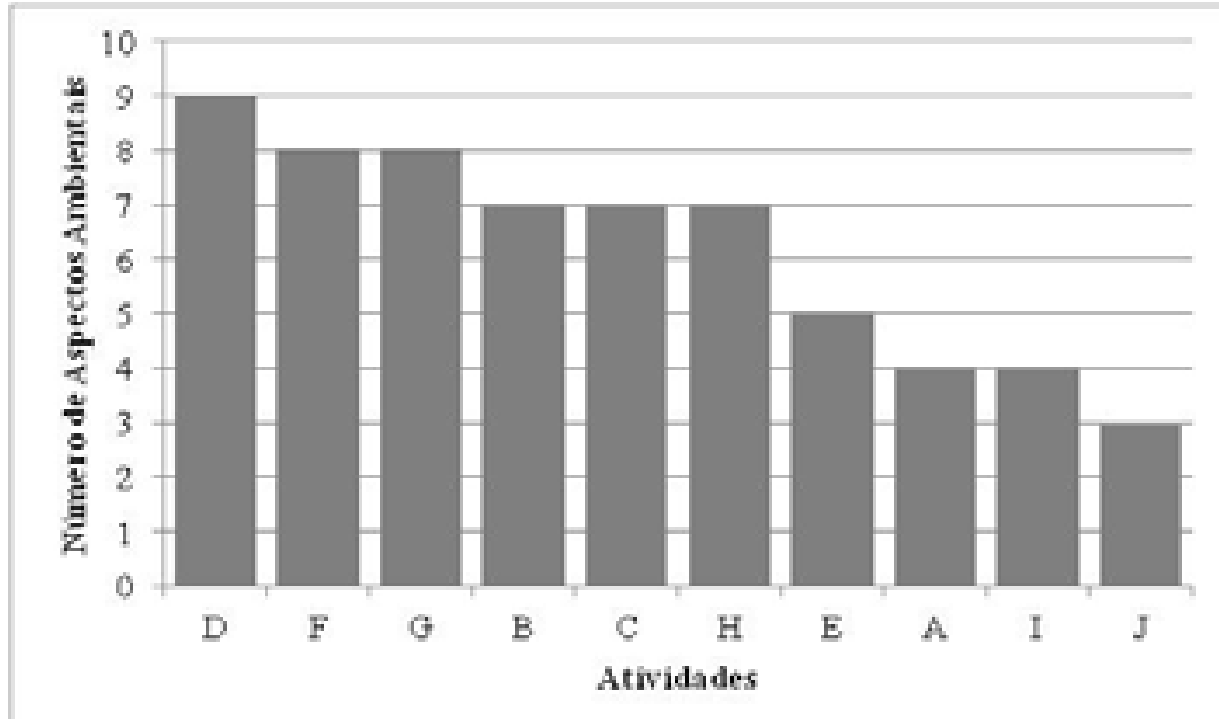

(a) Ordenação das atividades em função dos aspectos e impactos ambientais 


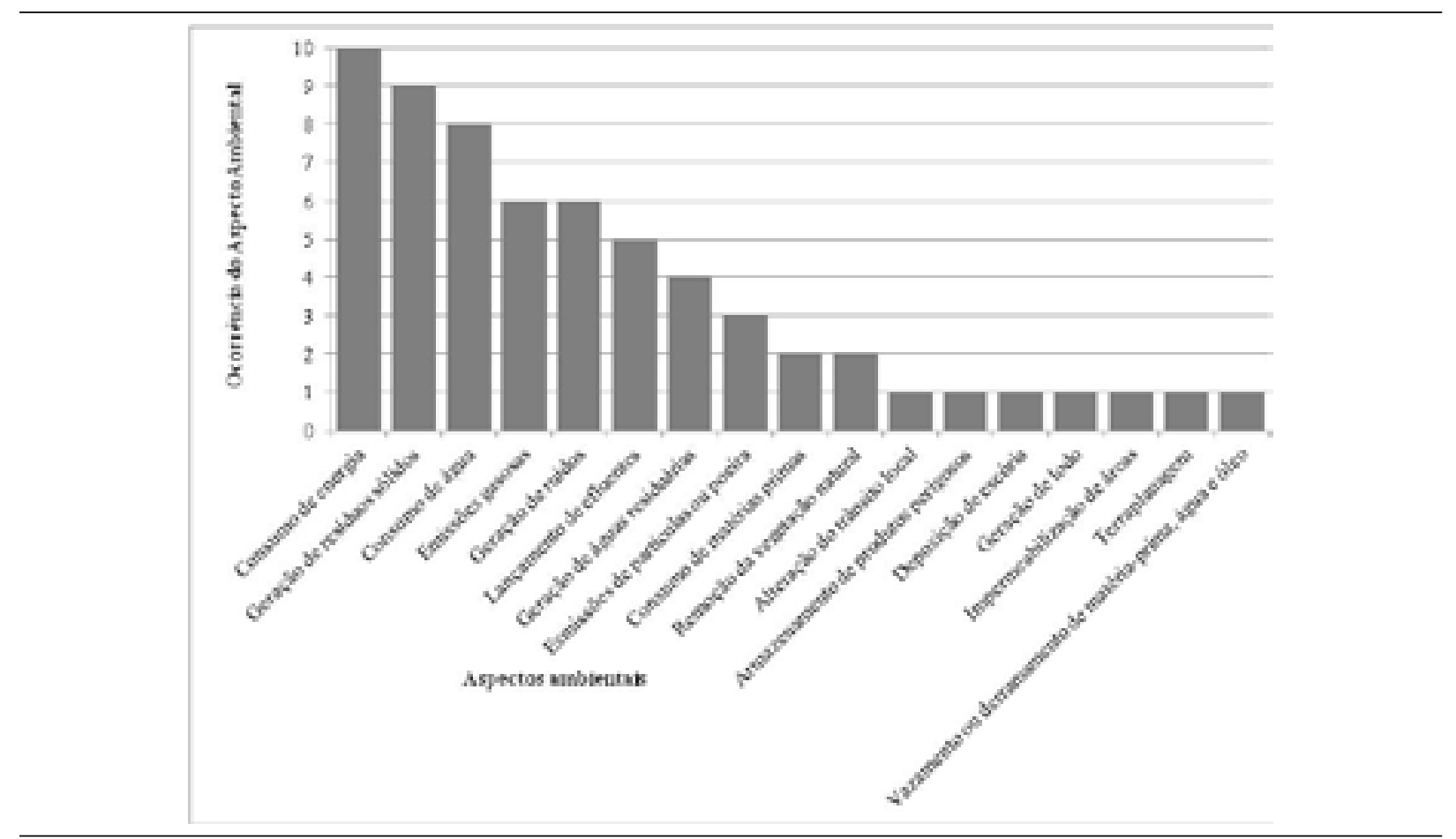

(b) Ordenação dos aspectos ambientais de acordo com o número de ocorrência no contexto das 10 atividades analisadas

Figura 7- Atividades vs aspectos e impactos ambientais (relacionar com a tabela 3).

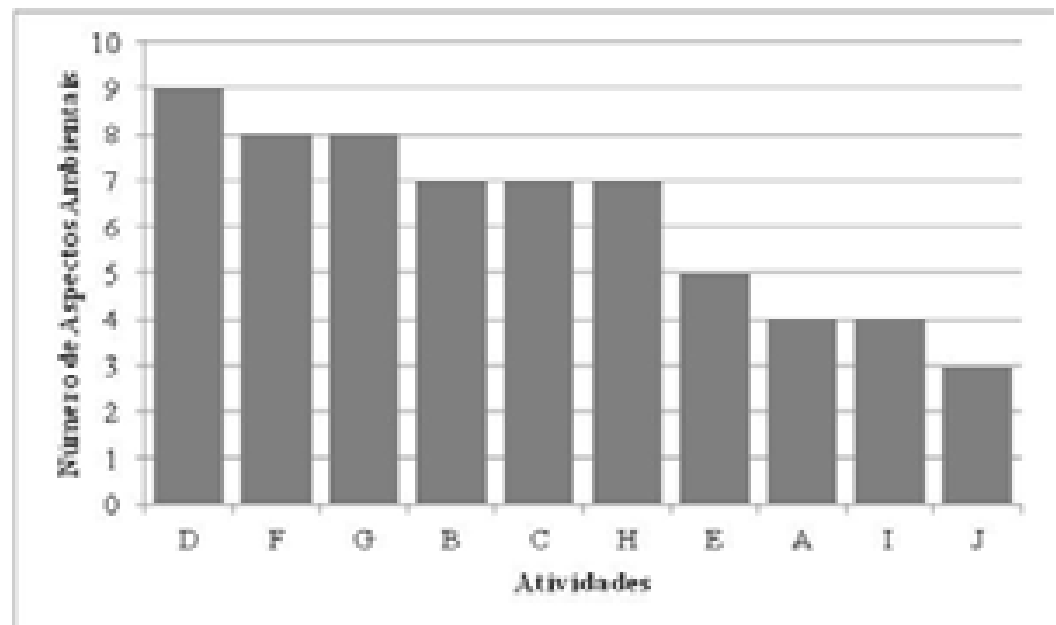

(a) Ordenação das atividades em função dos aspectos e impactos ambientais 


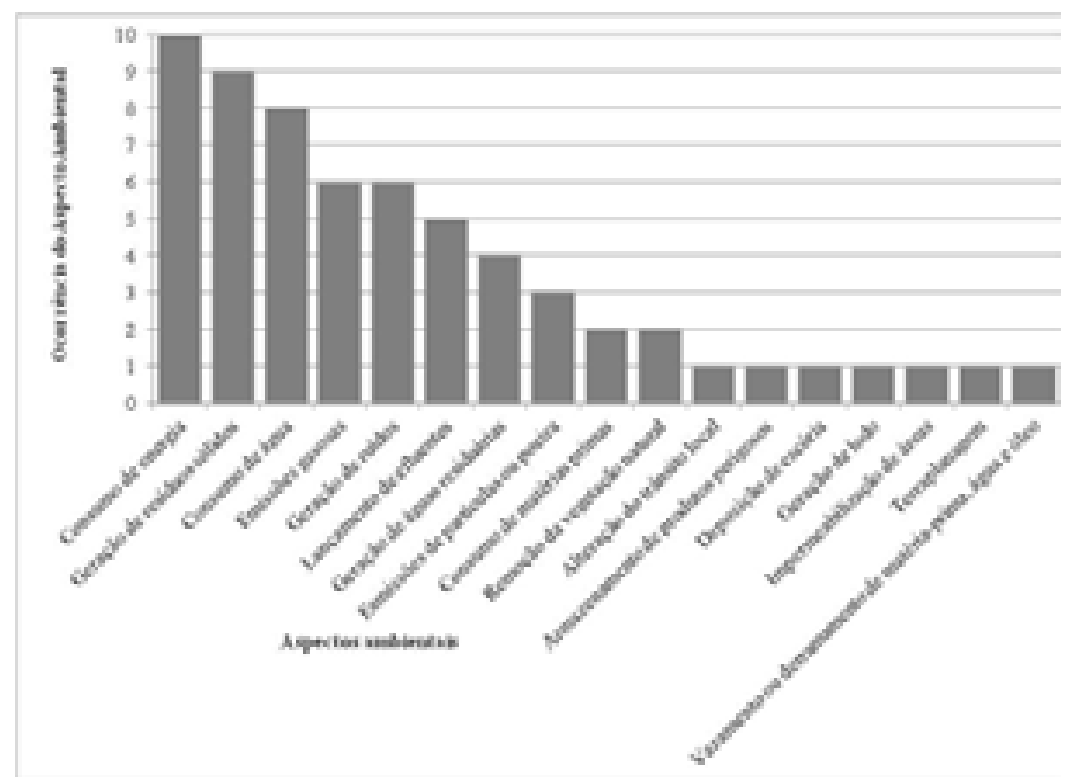

(a) Ordenação dos aspectos ambientais de acordo com o número de ocorrência no contexto das 10 atividades analisadas

As organizações dos setores primário e secundário têm um papel determinante no âmbito dos desafios impostos pela sustentabilidade, designadamente no que respeita à (1) conservação dos ecossistemas e (2) proteção social. Assim, espera-se uma crescente demonstração do sentido de responsabilidade ambiental e social das organizações empresariais, em conformidade com os objetivos do desenvolvimento sustentável para 2030 (UNITED NATIONS, 2015), através de medidas que visem:

garantir a conservação dos serviços dos ecossistemas (oferecidos através do solo, água, atmosfera e biota) através da redução substancial dos seus aspectos e impactos ambientais significativos. É oportuno salientar que as organizações, sobretudo as dos setores primário e secundário, têm um grande contributo a dar no domínio do desenvolvimento sustentável porque exercem um impacto significativo (direto e indireto) na depleção dos recursos naturais (incluindo a biodiversidade) e na poluição do solo, água e atmosfera (através de emissão de gases de estufa) e na saúde e bem-estar das populações;

promover a proteção social aumentando o nível de responsabilidade social à escala local, regional e/ou global mediante um envolvimento participativo e proativo nas ações sociais de combate à pobreza, desigualdade, desemprego e precariedade das condições sanitárias e de saúde. 


\section{Tabela 4 - Aspectos e impactos ambientais relacionados às organizações do sector secundário}

\begin{tabular}{|c|c|c|c|c|}
\hline $\begin{array}{l}\text { Código } \\
\text { NACE }\end{array}$ & Atividade & $\begin{array}{l}\text { Aspectos } \\
\text { ambientais }\end{array}$ & $\begin{array}{l}\text { Impactos } \\
\text { ambientais }\end{array}$ & $\begin{array}{l}\text { Medidas de mitigação mais } \\
\text { comuns }\end{array}$ \\
\hline \multirow{6}{*}{$\begin{array}{l}\overrightarrow{0} \\
0 \\
0 \\
0\end{array}$} & \multirow{2}{*}{$\begin{array}{l}\text { Operações da } \\
\text { planta industrial }\end{array}$} & $\begin{array}{c}\text { Lançamento de } \\
\text { efluentes (incluindo } \\
\text { águas de lavagem, } \\
\text { lixiviação das áreas de } \\
\text { depósitos de materiais } \\
\text { ou rejeitos) e águas de } \\
\text { resfriamento. }\end{array}$ & \multirow[t]{2}{*}{$\begin{array}{l}\text { Poluição de recursos } \\
\text { hídricos e edáficos } \\
\text { (relativos ao solo) }\end{array}$} & $\begin{array}{c}\text { Inclusão de sistemas de } \\
\text { drenagem em depósitos de } \\
\text { materiais para evitar a lixiviação } \\
\text { e a contaminação das águas } \\
\text { pluviais } \\
\text { Impermeabilização de áreas de } \\
\text { armazenamento } \\
\text { Controle na fonte: tratamento } \\
\text { de efluentes líquidos. }\end{array}$ \\
\hline & & $\begin{array}{c}\text { Emissões de } \\
\text { partículas para a } \\
\text { atmosfera } \\
\text { Emissões gasosas de } \\
\text { óxidos de enxofre e } \\
\text { nitrogênio, amoníaco, } \\
\text { névoas ácidas e } \\
\text { compostos de flúor. }\end{array}$ & & $\begin{array}{l}\text { Controle de emissões com o uso } \\
\text { de equipamentos como ciclones, } \\
\text { filtros de manga, precipitadores } \\
\text { eletrostáticos e lavadores, entre } \\
\text { outros. }\end{array}$ \\
\hline & \multirow[b]{2}{*}{$\begin{array}{l}\text { Armazenamento } \\
\text { de produtos } \\
\text { perigosos }\end{array}$} & \multirow{2}{*}{$\begin{array}{l}\text { Liberação casual de } \\
\text { solventes e materiais } \\
\text { ácidos ou alcalinos, } \\
\text { potencialmente } \\
\text { perigosos. }\end{array}$} & \multirow{2}{*}{$\begin{array}{l}\text { Poluição do espaço } \\
\text { biofísico com } \\
\text { o consequente } \\
\text { comprometimento } \\
\text { dos serviços dos } \\
\text { ecossistemas** } \\
\end{array}$} & $\begin{array}{c}\text { Manutenção preventiva das } \\
\text { áreas de armazenamento. }\end{array}$ \\
\hline & & & & $\begin{array}{c}\text { Instalação de diques e bacias ao } \\
\text { redor ou a jusante dos tanques } \\
\text { de armazenamento de produtos } \\
\text { perigosos. }\end{array}$ \\
\hline & $\begin{array}{l}\text { Geração de } \\
\text { resíduos }\end{array}$ & $\begin{array}{l}\text { Disposição de } \\
\text { resíduos sólidos. }\end{array}$ & $\begin{array}{l}\text { Contaminação } \\
\text { do solo e/ou } \\
\text { águas superficial e } \\
\text { subterrânea }\end{array}$ & $\begin{array}{c}\text { Redução, reutilização e } \\
\text { reciclagem dos subprodutos } \\
\text { numa perspectiva de } \\
\text { prolongamento do ciclo de vida } \\
\text { Tratamento adequado, antes da } \\
\text { disposição final, dos resíduos } \\
\text { sólidos, cujo reaproveitamento } \\
\text { não seja viável } \\
\text { Na impossibilidade do } \\
\text { tratamento, deverão ser } \\
\text { armazenados de maneira } \\
\text { adequada. }\end{array}$ \\
\hline & $\begin{array}{c}\text { Operação } \\
\text { industrial e } \\
\text { transporte } \\
\text { (incluindo carga } \\
\text { e descarga) }\end{array}$ & $\begin{array}{l}\text { Operações que geram } \\
\text { ruídos }\end{array}$ & Poluição sonora & $\begin{array}{c}\text { Tratamento acústico, } \\
\text { por exemplo, através } \\
\text { do enclausuramento de } \\
\text { equipamentos ou da proteção } \\
\text { acústica nas edificações que } \\
\text { abrigam os equipamentos } \\
\text { ruidosos ou ainda nas unidades } \\
\text { cujas operações produzam } \\
\text { ruídos acima do nível legalmente } \\
\text { aceitável. }\end{array}$ \\
\hline
\end{tabular}

*Medidas de mitigação em observância aos princípios de gestão do ambiente: precaução, prevenção, redução na fonte, participação, integração, adaptabilidade, gestão adaptativa, entre outros.

${ }^{* *}$ Os serviços de ecossistemas são fornecidos diretamente pelos principais componentes do ecossistema: solo, água, vegetação e biota, ou indiretamente através das suas interações, cuja complexidade estamos longe de dominar. 


\section{SÍNTESE FINAL}

Analisou-se comparativamente a situação da gestão ambiental e da qualidade através de indicadores referentes à evolução da produção científica e certificação das empresas.

Quanto à evolução das empresas certificadas, merece destaque que os resultados indicam uma evolução crescente da atribuição dos certificados nas diferentes unidades federativas (UF), ao longo dos anos. Complementarmente, constatou-se que: (i) não existe diferença significativa entre o número de produção científica nas áreas da gestão ambiental (ISO 14001) e da gestão da qualidade (ISO 9001). Contudo, existe uma assimetria na distribuição mundial estando concentradas na América do Norte (EUA e Canadá) e Europa; (ii) apesar de a comunidade científica brasileira estar produzindo, continuamente, conhecimento referente à gestão do ambiente com base na ISO 14001, em maior número comparativamente à gestão da qualidade (ISO 9001), a realidade empresarial é bem diferente, porquanto o número de empresas com a certificação ISO 14001 corresponde a apenas 6,6\% das que possuem certificação ISO 9001. Este fato pode estar relacionado com os maiores custos associados à manutenção de um sistema de gestão ambiental. Quando se efetua a análise com base nas unidades federativas, constata-se que, do total das empresas certificadas a nível nacional de acordo com ISO 14001 e 9001,39\% e 33\%, se localizam em São Paulo, respectivamente. Os estados de Santa Catarina e Paraná estão na segunda posição, com cerca de $16 \%$ de empresas certificadas.

É necessário promover a implementação dos sistemas de gestão (ambiental e da qualidade) nas empresas visto que contribuem para inventariação e monitoramento dos aspectos e impactos ambientais das atividades espacialmente distribuídas e que, por sua vez, fornecem dados/ informações que subsidiam o planejamento do território e gestão ambiental.

\section{REFERÊNCIAS}

ÁVILA, L.V.; HOFFMANN, C.; CORRÊA, A.C.; MADRUGA, L.R.; SCHUCH JÚNIOR, V.F.; SOUSA JÚNIOR, A.F.; ZANINI, R.R. Social Responsibility Initiatives Using ISO 26000: An Analysis from Brazil. Environmental Quality Management, v. 23, n. 2, p. 15-30, 2013.

BARCZAK, R.; DUARTE, E. Impactos Ambientais da Mobilidade Urbana: Cinco Categorias de Medidas Mitigadoras. URBE-Revista Brasileira de Gestão Urbana (Brazilian Journal of Urban Management), v. 4, n. 1, p. 13-32, 2012.

BRUNELLE-YEUNG, E.; MASEK, T.; ROJO, J.J.; LEVY, J.; ARUNACHALAM, S.; MILLER, S.M.; BARRET, S.R.H.; KUHN, S.R.;WAITZ, I.A. Assessing the Impact of Aviation Environmental Policies on Public Health. Transport Policy, v. 34, p. 21-28, 2014.

CROSSMAN, N.D.; BRYAN, B.A.; GROOT, R.S.; LIN, Y.; MINANG, P.A. Land Science Contributions to Ecosystem Services. Current Opinion In Environmental Sustainability, v. 5, p. 509-514, 2013. 
DJEKIC, I.; RAJKOVIC, A.; TOMIC, N.; SMIGIC, N.; RADOVANOVIC, R. Environmental Management Effects in Certified Serbian Food Companies. Journal of Cleaner Production, v. 76, p. 196-199, 2014.

HAIDER,J.; HASHMI, M.S.J. Health and Environmental Impacts in Metal Machining Processes. Comprehensive Materials Processing, v. 8 (Health, Safety and Environmental Issues), p. 7-33, 2014.

GRIGORATOS, T.; SAMARA, C.; VOUTSA, D.; MANOLI, E.; KOURAS, A. Chemical Composition and Mass Closure of Ambient Coarse Particles at Traffic and Urban-background Sites in Thessaloniki, Greece. Environmental Science and Pollution Research, v. 21, n- 12, p. 7708-7722, 2014.

GÜNTHER,W.M.R. Áreas Contaminadas no Contexto da Gestão Urbana. São Paulo em Perspectiva, v. 20, n. 2, p. 105-117, 2006.

JABBOUR, A.B.L.S.; JABBOUR, C.J.; LATAN, H.; TEIXEIRA, A.A.; OLIVEIRA, J.H.C. Quality Management, Environmental Management Maturity, Green Supply Chain Practices and Green Performance of Brazilian Companies with ISO 14001 Certification: Direct and Indirect Effects. Transportation Research Part E, v. 67, p. 39-51, 2014.

JACOBI, P.R.; BESEN, G.A.R. Solid Waste Management in São Paulo: The challenges of sustainability. Estudos Avançados, v. 25, n. 71, p. 135-158, 2011.

KONDO, M.C.; GROSS-DAVIS, C.A.; MAY, K.; DAVIS, L.O.; JOHNSON, T.; MALLARD, M.; GABBADON, A.; SHERROD, C.; BRANAS, C.C. Place-based Stressors Associated With Industry and Air Pollution. Health \& Place, v. 28, p. 31-37, 2014.

LEME, M.M.V.; ROCHA, M.H.; LORA, E.E.S; VENTURINI, O.J.; LOPES, B.M.; FERREIRA, C.H. Techno-Economic Analysis and Environmental Impact Assessment Of Energy Recovery From Municipal Solid Waste (MSW) In Brazil. Resources, Conservation and Recycling, v. 87, p. 8-20, 2014.

LOISEAU, E.; JUNQUA, G.; ROUX, P.; BELLON-MAUREL, V. Environmental Assessment of a Territory: An Overview of Existing Tools and Methods. Journal of Environmental Management, v. 112, p. 213-225, 2012.

MCKENZIE, K.; MURRAY, A.; BOOTH, T. Do urban environments increase the risk of anxiety, depression and psychosis? An Epidemiological Study. Journal of Affective Disorders, v. 150, n. 3, p. 1019-1024, 2014.

MOLINA-AZORÍN,J.F.;TARÍ,J.J.; PEREIRA-MOLINER,J.; LÓPEZ-GAMERO, M.D.; PERTUSA-ORTEGA, E.M. The Effects of Quality and Environmental Management on Competitive Advantage: A Mixed Methods Study in the Hotel Industry. Tourism Management, v. 50, p. 41-54, 2015. 
NEAL, C.; HOUSE,W.A.;JARVIE,H.P.; LEEKS, G.J.L.; MARKER, A.H.Conclusions to the special volume of science of the total environment concerning UK fluxes to the North Sea, land ocean interaction study river basins research, the first two years. Science of The Total Environment, v. 194-195, p. 467-477, 1997.

OLIVEIRA, O.J.; SERRA, J.R.; SALGADO, M.H. Does ISO 14001 Work in Brazil? Journal of Cleaner Production, v. 18, n. 18, p. 1797-1806, 2010.

OMAR, D.; KARUPPANAN, S.; AYUNISHAFIEA, F. Environmental Health Impact Assessment of a Sanitary Landfill in An Urban Setting. Procedia-Social and Behavioral Sciences, v. 68, p. 146-155, 2012.

PALMER, M.A.; BERNHARDT, E.S.; CHORNESKY, E.A. et al. Ecological Science and Sustainability for the $21^{\text {st }}$ Century. Frontiers in Ecology and the Environment, v. 3, n. 1, p. 4-11, 2005.

POMBO, F.R.; MAGRINI, A. Panorama de Aplicação da Norma ISO 14001 no Brasil. Gestão \& Produção, v. 15, n. 1, p. 1-10, 2008.

REHFUESS, E.A.; BARTRAM, J. Beyond direct impact: Evidence synthesis towards a better understanding of effectiveness of environmental health interventions. International Journal of Hygiene and Environmental Health, v. 217, n. 2-3, p. 155-159, 2014.

SABOIA, J. A Continuidade do Processo de Desconcentração Regional da Indústria Brasileira nos Anos 2000. Nova Economia, v. 23, n.2, p. 219-278, 2013.

SILVA, V.P.R.; ALEIXO, D.O.; ALMEIDA, R.S.R.; CAMPOS, J.H.B.C.; ARAÚJO, L.E. Modelo integrado das pegadas hídrica, ecológica e de carbono para o monitoramento da pressão humana sobre o planeta. Ambiência, v. 11, n. 3, p. 639-639, 2015.

SU, S.; XIAO, R.; MI, X.; XU,X.; ZHANG,Z.;WU,J. Spatial determinants of hazardous chemicals in surface water of Qiantang River, China. Ecological Indicators, v. 24, p. 375-381, 2013.

TESTA, F.; RIZZI, F.; DADDI, T.; GUSMEROTTI, N.M.; FREY, M.; IRALDO, F. EMAS and ISSO 14001: the Differences in Effectively Improving Environmental Performance. Journal of Cleaner Production, v. 68, p. 165-173, 2014.

TUCCI, C.E.M. Águas Urbanas. Estudos Avançados, v. 22, n. 3, p. 97-112, 2008.

UNITED NATIONS. Transforming our world: the 2030 agenda for sustainable development. A/RES/70/1. New York: United Nations, 2015.35p.

WANG, Y.; BAKKER, F.; GROOL, R.; WORTCHE, H. Effect of ecosystem services provided by urban green infrastructure on indoor environment: a literature review. Building and Environment, 77, 88-100, 2014. 